\title{
Communication \\ Feasibility of Probing the Filler Restructuring in Magnetoactive Elastomers by Ultra-Small-Angle Neutron Scattering
}

\author{
Inna A. Belyaeva ${ }^{1}$, Jürgen Klepp ${ }^{2, *(\mathbb{D}}$, Hartmut Lemmel ${ }^{3,4}$ and Mikhail Shamonin $^{1}$ (1) \\ 1 East Bavarian Centre for Intelligent Materials (EBACIM), Ostbayerische Technische Hochschule (OTH) \\ Regensburg, Prüfeninger Str. 58, 93049 Regensburg, Germany; inna.belyaeva@oth-regensburg.de (I.A.B.); \\ mikhail.chamonine@oth-regensburg.de (M.S.) \\ 2 Faculty of Physics, University of Vienna, Boltzmanngasse 5, 1090 Vienna, Austria \\ 3 Atominstitut, Technische Universität Wien, 1020 Vienna, Austria; lemmel@ill.fr \\ 4 Institut Laue-Langevin, 38042 Grenoble, France \\ * Correspondence: juergen.klepp@univie.ac.at
}

Citation: Belyaeva, I.A.; Klepp, J.; Lemmel, H.; Shamonin, M. Feasibility of Probing the Filler Restructuring in Magnetoactive Elastomers by UltraSmall-Angle Neutron Scattering. Appl. Sci. 2021, 11, 4470. https://doi. org/10.3390/app11104470

Academic Editor: Sebastian Jaksch

Received: 12 April 2021

Accepted: 10 May 2021

Published: 14 May 2021

Publisher's Note: MDPI stays neutral with regard to jurisdictional claims in published maps and institutional affiliations.

Copyright: (c) 2021 by the authors. Licensee MDPI, Basel, Switzerland. This article is an open access article distributed under the terms and conditions of the Creative Commons Attribution (CC BY) license (https:// creativecommons.org/licenses/by/ $4.0 /)$.

\begin{abstract}
Ultra-small-angle neutron scattering (USANS) experiments are reported on isotropic magnetoactive elastomer (MAE) samples with different concentrations of micrometer-sized iron particles in the presence of an in-plane magnetic field up to $350 \mathrm{mT}$. The effect of the magnetic field on the scattering curves is observed in the scattering vector range between $2.5 \times 10^{-5}$ and $1.85 \times$ $10^{-4} \AA^{-1}$. It is found that the neutron scattering depends on the magnetization history (hysteresis). The relation of the observed changes to the magnetic-field-induced restructuring of the filler particles is discussed. The perspectives of employing USANS for investigations of the internal microstructure and its changes in magnetic field are considered.
\end{abstract}

Keywords: ultra-small-angle neutron scattering; magnetoactive elastomer; magnetorheological elastomer; hysteresis; restructuring of the filler

\section{Introduction}

Magnetoactive elastomers (MAEs) can be classified as magnetic composite materials, i.e., polymer matrices filled with magnetic micro-particles. The growing interest in MAEs is determined by the possibility of controlling their mechanical and other physical properties by application of technically easy realizable magnetic fields [1-9]. The physical reason for the observed changes in MAE properties are field-induced reconfigurations of the microstructure formed by the magnetic particles [1,5,10-12], which are possible in mechanically compliant polymer matrices [8]. These rearrangements of filling particles in an externally applied magnetic field have been observed directly by optical [13] and X-Ray methods $[14,15]$.

The internal filler structure of nanocomposites with magnetic particles has been studied by small-angle neutron scattering (SANS) in the past [16]. Hitherto, most of SANS research has concentrated on ferrofluids [16-19] which are highly dispersed systems of nanometer-sized ferromagnetic or ferrimagnetic particles in a liquid medium (organic or inorganic fluids, water). Recently, first papers appeared, where magnetizable nano- [20-23] and micro-particles [21] embedded into an elastomer matrix have been studied by SANS in the scattering vector $Q$ range $\sim 0.01-0.1 \AA^{-1}$. Such scattering vectors do not correspond to the size of filling micro-particles. Balasiou et al. [21] have found that the SANS scattering intensities of an elastomer matrix with iron $(\mathrm{Fe})$ micro-particles exhibited power law behaviour $I(Q) \approx Q^{-\alpha}$ with the exponent $3<\alpha<4$. For elastomer samples with $\mathrm{Fe}$ particle concentration between 25 and 75 mass $\%$, a surface fractal dimension of $D_{s} \approx 2.2$ was obtained. The measurements were performed in the absence of a magnetic field. A theoretical approach to extract information about the internal structure of magnetic filament brushes from scattering experiments has been recently proposed by Pyanzina et al. [24]. 
Borin et al. [25] developed a Small Angle Light Scattering (SALS) laboratory setup with simultaneous exposure of fluidic samples to a shear flow and an external magnetic field and used it for investigation of structuring processes in a magnetic fluid comprising clustered iron oxide nanoparticles. They observed an anistropy of the scattering patterns in an applied magnetic field. Zákutná et al. [26] reported a magnetorheological SANS setup at the Institut Laue-Langevin allowing for an in-situ magnetic field up to $20 \mathrm{mT}$ and the shear flow of ferrofluids. The maximum magnetic field in this setup is one order of magnitude below the magnetic-field values required to observe the effects caused by magnetic-field restructuring in MAEs.

Conventional SANS is an experimental technique that uses elastic neutron scattering at small scattering angles to investigate the structure of various substances at a mesoscopic scale of about 1-100 nm. Therefore, it has been neglected for investigation of MAEs, where micro-particles are employed. In the present paper, we use the ultra-small-angle neutron scattering (USANS) instrument S18 at the Institut Laue-Langevin where the length scale that can be analyzed is extended beyond $10 \mu \mathrm{m}$.

The displacements of magnetic particles in MAEs can be observed by using X-ray micro-computed topography, but this method becomes unreliable at high concentrations of filler particles due to X-ray absorption. It is known that SANS allows one to determine the fractal organization of a cluster (mass fractal) because a certain correlation between particles occurs in the case of their aggregation. It is also largely accepted that the reduction of the shear storage modulus of filled elastomers with the growing amplitude of shearing oscillations (known as the Payne effect) in nanocomposites can be attributed to the agglomeration/de-agglomeration of filler-particle aggregates and is determined by the fractal dimension of the cluster. Very recently, it was hypothesized that USANS may be used to determine the magnetic-field-induced changes of fractal dimension of aggregates of ferromagnetic particles in MAEs [27]. In general, it can be expected that if a rearrangement of magnetized inclusions takes place in a magnetic field, such a change in a microstructure should be observable in a USANS experiment if the size of particles or their aggregates is comparable with the magnitude of the observable scattering vector. Given some similarity between operation modes of MAEs and magnetic fluids, it would be interesting to obtain additional information on the internal microstructure of MAEs using neutron scattering techniques, as it has been successfully done in magnetic fluids. This Communication reports the first USANS observation in MAEs in the scattering vector range $2.5 \times 10^{-5}-1.85 \times 10^{-4} \AA^{-1}$ (corresponding to structure sizes between $25 \mu \mathrm{m}$ and $3.4 \mu \mathrm{m}$ ) and the effect of magnetic fields on the scattering curves.

\section{Materials and Methods}

\subsection{Samples}

Six thin-film MAE samples have been synthesized. The fabrication of MAE samples is described in detail in $[27,28]$. All samples have been cross-linked in the absence of a magnetic field and, therefore, it is known that they can be considered isotropic. The soft-magnetic carbonyl iron powder (CIP) type SQ (mean particle size d50 of $4.5 \mu \mathrm{m}$, no coating), provided by BASF SE Carbonyl Iron Powder \& Metal Systems (Ludwigshafen, Germany), was used as the ferromagnetic filler.

Table 1 presents the iron content in three samples that had a soft elastomer as a matrix (the shear storage modulus is $\sim 1 \mathrm{kPa}$ ). The thickness of MAE samples was $0.3 \pm 0.1 \mathrm{~mm}$. The thickness of the matrix (empty) sample was $0.7 \mathrm{~mm}$. Measurements of SANS scattering curves of the matrix in the absence as well as in the presence of a magnetic field did not reveal any significant effect of magnetic field on the course of curves. We also investigated three MAE samples with 60 and 67 mass $\%$ of Fe which have been fabricated with a stiffer polymer matrix (the shear storage modulus is $\sim 10 \mathrm{kPa}$ ). There one may expect that the magnetic-field restructuring will be diminished by a stiffer matrix. 
Table 1. Samples.

\begin{tabular}{ccc}
\hline Sample & Mass Fraction of Fe, $\%$ & Volume Fraction of Fe, $\%$ \\
\hline Matrix & 0 & 0 \\
MAE10 & 10 & 1.3 \\
MAE30 & 30 & 4.9 \\
MAE80 & 80 & 32.4 \\
\hline
\end{tabular}

\subsection{Measurements}

The USANS data were measured at the S18 interferometry and USANS facility of the Institut Laue-Langevin, Grenoble, France (Figure 1).

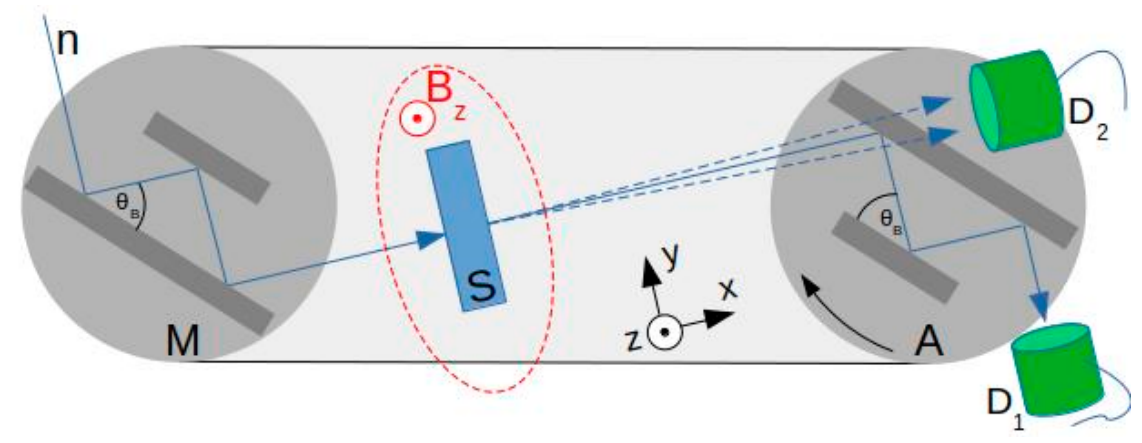

Figure 1. USANS setup (not to scale): so-called Bonse-Hart camera configuration with channel-cut silicon perfect crystals (monochromator $\mathrm{M}$ and analyzer $\mathrm{A}$, lattice planes are parallel to the crystal slab faces), sample $S$ in a region with an applied vertical, static magnetic field $B_{z}$, and detectors $D_{1}$ and $\mathrm{D}_{2}[29]$.

The non-polarized neutron beam from the top left is diffracted by $\mathrm{M}$ to pass the sample $S$ at normal incidence. Portions of the beam that are scattered by $S$ will be diffracted by analyzer $A$ and detected by detector $D_{1}$ at angular positions of $A$ slightly different from the Bragg position $\theta_{\mathrm{B}}$. The accuracy of the piezo system controlling A's angular position is $0.036^{\prime \prime}$. The $Q$-values in our USANS experiments refer to variation of A's angular position and are calculated from measured absolute piezo encoder values. Lowbackground conditions and the excellent suppression of diffraction-peak side lobes enable us to perform measurements at $Q$-values as small as a few $10^{-5} 1 / \AA$. The wavelength of the incident beam was $1.9 \AA$. Neutrons are counted in detectors $D_{1}$ and $D_{2}$ as a function of angular position of $\mathrm{A}$ with and without sample. The values are normalized by the orders of magnitude larger count rate at a monitor detector upstream to account for power fluctuations of the neutron source (reactor) during the measurement. The scattering curve measured without sample was interpolated and subtracted from the curves measured with samples.

The dc magnetic field is applied vertically (z-direction) and it is in the plane of MAE samples. It can be varied from 0 to $350 \mathrm{mT}$. The neutron beam is directed perpendicular to the sample's plane and to the magnetic field vector. No visible deformation of MAE samples in the maximum magnetic field has been observed. The magnitude of the scattering vector $Q$ refers to the horizontal plane. It can be expected that the restructuring (formation of elongated aggregates) occurs in the vertical direction and we mainly observe the scattering in the perpendicular direction. Unfortunately, due to the specific features of the USANS setup, it was not possible to record the 2D scattering patterns, because these measurements would be prohibitively long.

\section{Results and Discussion}

Although the data have been obtained in the $Q$-range up to $10^{-3} \AA^{-1}$, the following Figures show the data for the $Q$ values up of $1.85 \times 10^{-4} \AA^{-1}$. Even if we also suspect some magnetic-field-induced change in scattering intensity by structures smaller than 
$3.4 \mu \mathrm{m}\left(Q>1.85 \times 10^{-4} \AA^{-1}\right)$, we did not observe systematic and conclusive changes for larger $Q$ values. Figure 2 presents the USANS curves in zero, intermediate ( $200 \mathrm{mT})$ and maximum $(350 \mathrm{mT})$ magnetic field. There and in the following Figures the scattering curves are always normalized to the maximum of the presented curves (i.e., for a specific MAE sample). By such a normalization, the effect of magnetic field on neutron scattering in each particular MAE sample is demonstrated. We are currently not able to interpret the cross-correlation between scattering intensities of different samples, although such an information is available in the data set (cf. the data link at the end of this paper). The difference of the scattering curves is clearly seen and this difference grows with the increasing magnetic field. The total scattering intensity in the observed range of scattering vector is reduced with increasing magnetic flux density.

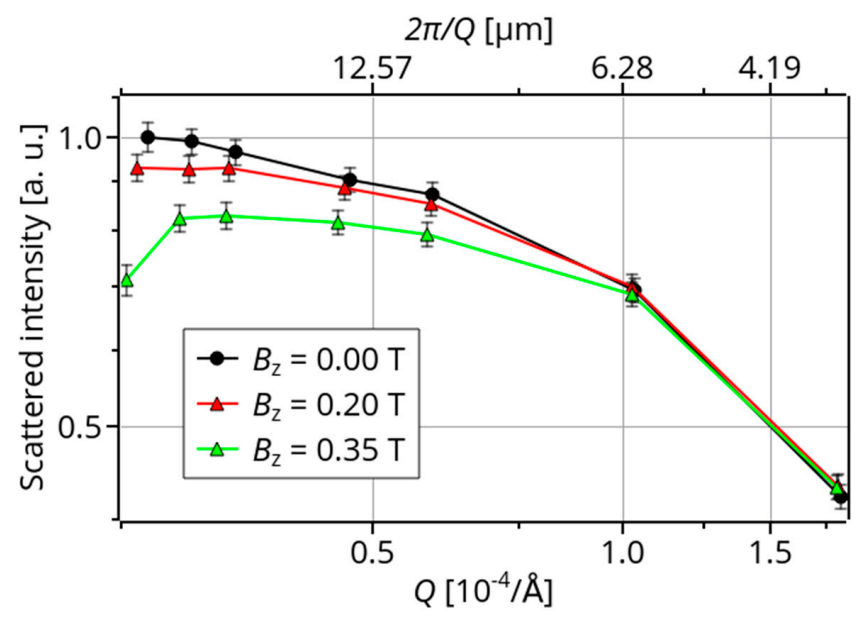

Figure 2. USANS curves of the MAE samples with 10 mass $\%$ of Fe for three different values of the magnetic flux density $B_{\mathrm{z}}$.

Figure 3 shows the USANS curves for the sample with 30 mass\% of Fe at different magnetic fields. Again, the effect of magnetic field is clearly observed. The scattering intensity becomes lower with the increasing magnetic field for $2.5 \times 10^{-5} \AA^{-1}<Q<$ $1 \times 10^{-4} \AA^{-1}$.

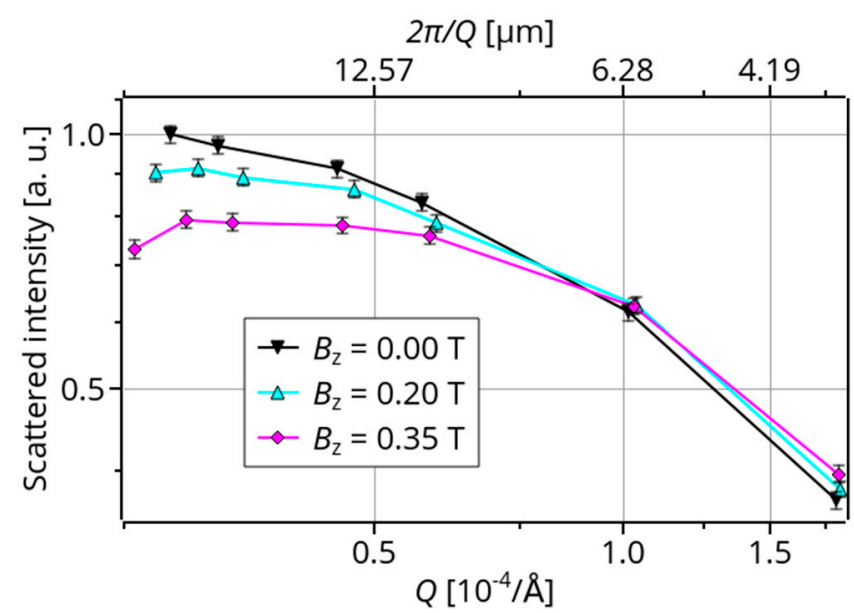

Figure 3. USANS curves of the MAE samples with 30 mass $\%$ of Fe for different magnetic fields.

As a control experiment, we measured the magnetic field dependence of the scattering curve of available MAE samples with the stiff matrix.

Figure 4 shows as an example the results for the MAE sample with 67 mass $\%$ of iron. A rather constant minor offset of the curve in the largest magnetic field appears to be within the measurement accuracy, so that no significant effect of magnetic field on the 
neutron scattering in the $Q$-range of interest is observed. In the current configuration both the nuclear and magnetic scattering cross sections are probed as $Q$ is perpendicular to the magnetization direction. However, if magnetic scattering was present in this case, we were not able to reliably separate its contribution applying rather low magnetic field and using the experimental configuration shown in Figure 1.

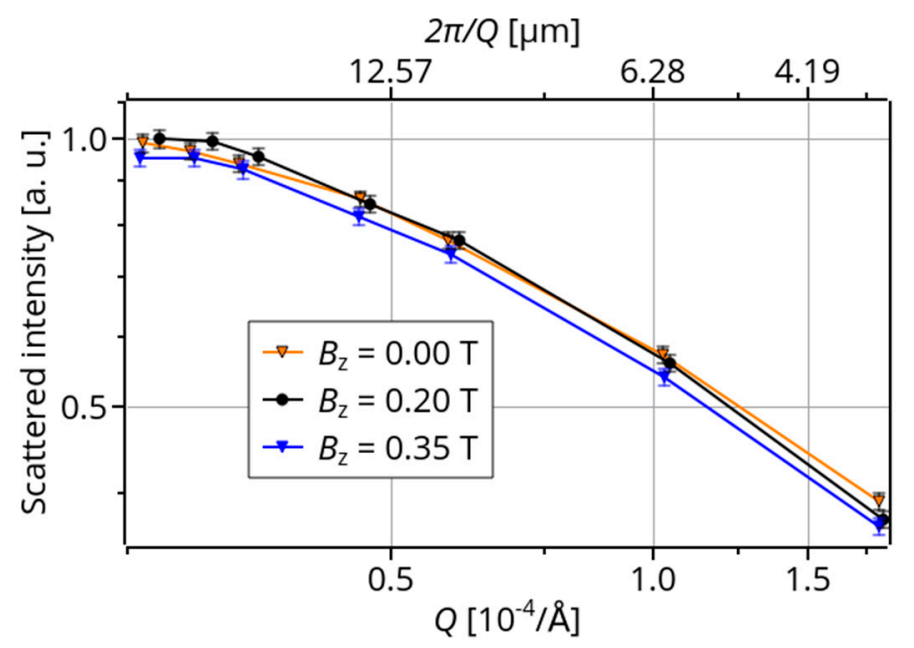

Figure 4. USANS curves of the MAE sample with 67 mass $\%$ of Fe and a stiff matrix for different magnetic fields.

Such an observation could be attributed to the limited mobility of filler micro-particles within the stiff matrix (as compared to the other MAE samples). The restructuring of filler particles is hindered in this case and the scattering behaviour is much less changed in comparison to other samples.

It is well known that physical properties of compliant MAE demonstrate hysteresis behaviour with respect to an applied magnetic field. The hysteresis occurs not only when the matrix is filled with hard-magnetic particles (where a kind of hysteresis has to be expected) but also for soft-magnetic inclusions with the vanishing magnetization reversal loop, which is the case in the present paper. Zubarev et al. [30] explained the hysteresis of magnetic properties by the hysteresis of the consolidation of filler particles into elongated aggregates. Since it is expected that the hysteresis is determined by different microstructures in MAEs depending on the magnetization history, it can be expected that the hysteresis can be observed in the scattering curves.

Figure 5 demonstrates the results of the scattering measurements. The following sequence of magnetic field strength was applied $0 \mathrm{~T}$ (denoted as (1) in Figure 5) $\rightarrow 0.2 \mathrm{~T}$ (2) $\rightarrow 0.35 \mathrm{~T}$ (3) $\rightarrow 0.2 \mathrm{~T}(4) \rightarrow 0 \mathrm{~T}(5) \rightarrow 0.2 \mathrm{~T}(6) \rightarrow 0.35 \mathrm{~T}(7) \rightarrow 0.2 \mathrm{~T}(8) \rightarrow 0 \mathrm{~T}(9)$. Indeed, the hysteresis of scattering curves is observed also in our USANS experiments. In particular, it is clearly seen that the three curves (1,5 and 9) obtained in the absence of a magnetic field are well separated from the family of curves obtained in the presence of a magnetic field. Moreover, these three curves do not coincide and they are progressively shifted towards the curves obtained in the presence of a magnetic field displaying the "memory" effect. Further, it is observed that the scattering curves 2 and 6 at $0.2 \mathrm{~T}$ corresponding to the ascending part of the hysteresis curve (i.e., when the field was increased from 0 to $0.2 \mathrm{~T}$ ) are distinctly different from the scattering curves 4 and 8 at $0.2 \mathrm{~T}$ when the field was decreased from $0.35 \mathrm{~T}$ to $0.2 \mathrm{~T}$ and at $0.35 \mathrm{~T}$. Interestingly, the scattering curves 4 and 8 seem to be similar to the curves 3 and 7 obtained in the maximum magnetic field. 


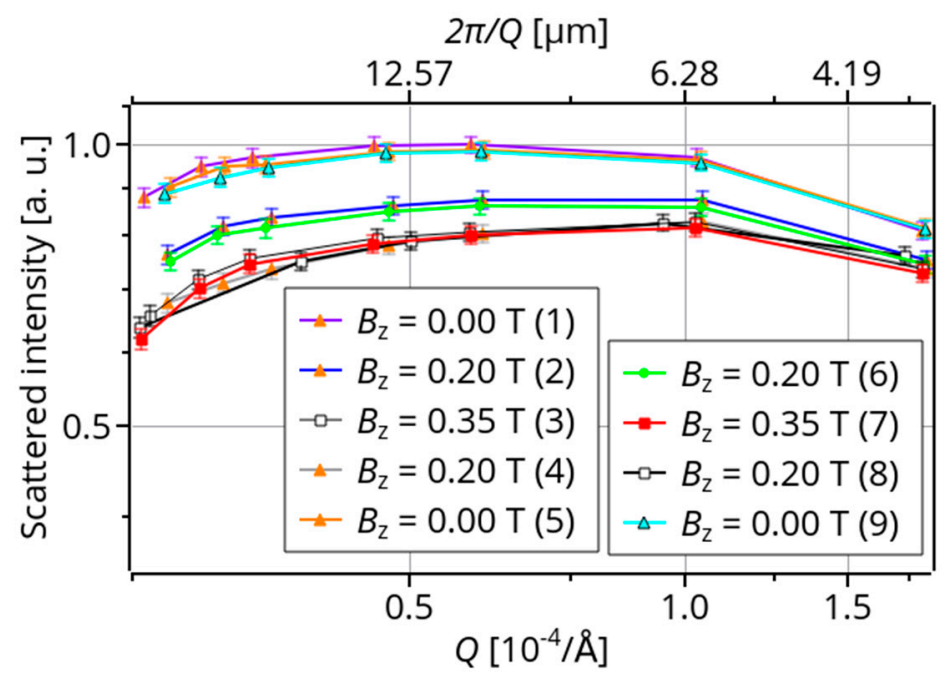

Figure 5. USANS curves of the MAE sample with 80 mass $\%$ of Fe and a soft matrix for different magnetic fields.

To qualitatively explain the observed differences in scattered curves, let us recall the known results on SANS scattering in a magnetic fluid [31]. The angle between the $Q$-vector and the magnetic field direction was set to be $0^{\circ}$ ( $Q$-vector parallel to the magnetic field direction), $30^{\circ}$ and $60^{\circ}$. In a magnetic fluid, the magnetic particles formed chain-like structures and a pronounced (Bragg) peak, corresponding to a resulting particle-particle distance was observed. The position of this peak was practically independent of the angle between the magnetic field vector and the $Q$-vector. From this one may conclude that for magnetic fluid, the inter-particle distance in chains determines the position of the scattering peak. In MAEs, the displacements of particles are limited by the matrix elasticity. For high concentrations of magnetizable particles in MAEs, numerical simulations showed that formation of chain-like structures may become impossible due to purely geometrical constraints [32]. Therefore, in MAEs it is more reasonable to speak about formation of elongated particle aggregates and not about chain formation. A physical picture of changes in internal MAE microstructure was proposed in [33]. Obviously, the arrangement of magnetic particle in an MAE becomes anisotropic. The corresponding physical properties, e.g., the (dielectric) permittivity become anisotropic as well [34]. We were able to observe the anisotropy of neutron scattering in MAE samples using a very-small-angle neutron scattering (VSANS) instrument, but there we could not avoid multiple scattering [35].

In our presented USANS experiments, the $Q$-vector is perpendicular to the magnetic field direction. We may speculate that typically structures larger than $25 \mu \mathrm{m}$ (elongated clusters) are formed in a highly filled sample ( 80 mass $\%$ of iron, see Figure 5$)$. For such a highly loaded MAE, the existence of the three-dimensional magnetic-filler network already in the absence of a magnetic field can be expected. Intuitively, one would anticipate that the structures would elongate in field direction and, thus, this dimension would grow, while perpendicular structures would rather shrink by such alignment. The presented data can be interpreted as a reduction of structure's size in the direction perpendicular to the field direction, and thus loss of scattering at lower $Q$. In Figure 5, the formation of a local maximum at $Q \approx 10^{-4} \AA^{-1}$ corresponding to the typical size of about $6 \mu \mathrm{m}$ is observed, which is close to the mean particle diameter. This would suggest the formation of elongated aggregates of aligned individual particles.

However, such explanation does not work well for Figures 2 and 3 where a tendency to formation of a local maximum corresponding to the typical size of roughly $20 \mu \mathrm{m}$ or larger can be assumed after what was said above. Also for these samples, loss of scattering for lower $Q$ is observed. The typical size of $20 \mu \mathrm{m}$ would be larger than the distance between uniformly distributed spherical particles with the mean diameter as used in our samples, corresponding to several particle diameters. Clearly, the particle concentration in Figures 2 
and 3 is much smaller than in Figure 5. It is unlikely that large particle aggregates formed in these samples in the non-magnetized state. The origin of the observed effects may be in the resulting anisotropy of neutron scattering due to magnetically induced anisotropy of particle distribution. Such an effect has been observed in [31] where it has been found that the maximum amplitude of scattering is in the direction parallel to the field direction.

The hysteresis of Figure 5 becomes more clearly visible in Figure 6 where we plotted the area under the interpolated scattering curve in dependence on the magnetic flux density. The points in Figure 6 have been obtained in the following way. Each curve in Figure 6 was linearly interpolated between the measurement points and then integrated, the result of integration was divided by the length of the $Q$ range and normalized by the maximum of the available results.

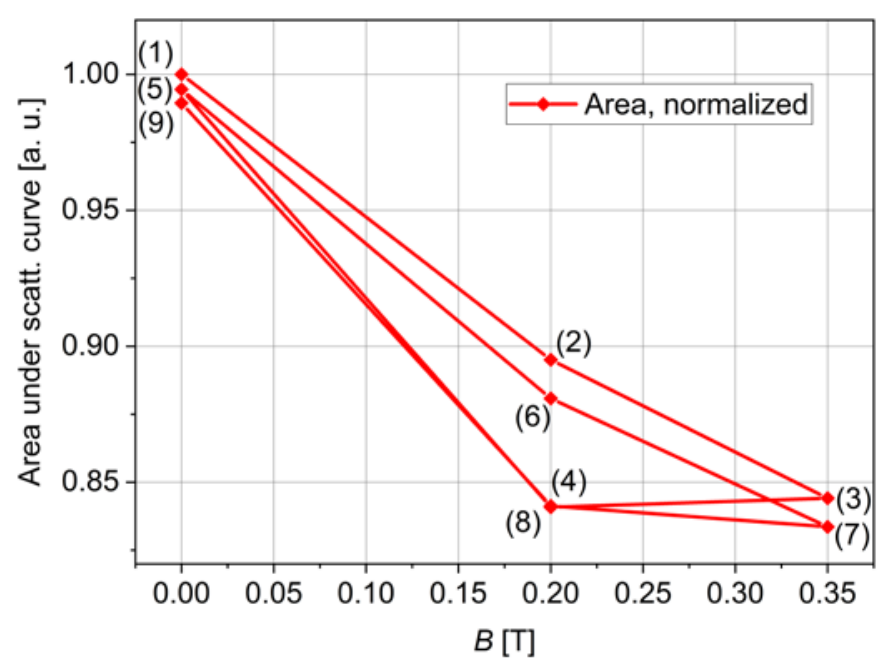

Figure 6. Normalized area under interpolated USANS scattering curve versus the applied magnetic field.

The hysteresis in Figure 6 is attributed to the magnetic-field-induced restructuring of filler particles, which is also the origin of magnetic hysteresis in such a material. It is known that the CIP particles are soft magnetic and possess a negligible magnetic hysteresis loop at room temperature [36]. In soft MAEs, the magnetic hysteresis appears in a particular form of a "pinched" hysteresis loop and it completely disappears at low temperatures when the matrix becomes stiff [37]. The relation of hysteresis of magnetic properties to the hysteresis of the consolidation of filler particles into elongated aggregates was explained by Zubarev et al. [30].

\section{Conclusions and Outlook}

Ultra-small-angle neutron scattering is a sensitive method for characterization of magnetoactive elastomers with micrometer-sized inclusions. We were able to observe the magnetic-field-induced changes in the scattering curves of soft MAEs in moderate magnetic fields of 200 and $350 \mathrm{mT}$. We believe that these changes are mainly due to modifications in the mutual arrangement of magnetic particles in a soft polymer matrix. It can be expected that the effect will be more pronounced in larger magnetic fields (say $600 \mathrm{mT}$ ), which should be verified in the future using another experimental setup. The USANS measurements confirm the previously put forward hypothesis that the hysteresis of physical properties of MAEs is related to the restructuring of filler particles.

To investigate the anisotropy of the field-direction-dependent (due to structural changes) nuclear neutron scattering and the contribution of magnetic neutron scattering, the experiments should be performed for different angles between the $Q$-vector and an applied magnetic field. This means that an experimental setup should allow for rotation of an electromagnet in the plane, which is perpendicular to the neutron beam. The usage of polarized neutron beams may be also helpful. 
USANS experiments at a low temperature $(<150 \mathrm{~K})$ would allow one to immobilize the filler particles in an elastomer matrix [37] and investigate the neutron scattering on "frozen" MAE samples in the absence of an external magnetic field.

The interpretation of the scattering curves remains an open question for future research. A suitable theory has to be developed for describing the scattering from the elongated agglomerates of micro-particles. We believe that the analysis of USANS on MAEs may shed light on the processes of restructuring of ferromagnetic filler particles in a magnetic field. Usage of smaller filler particles $(\approx 1 \mu \mathrm{m})$ may help to utilize the available $Q$-range more effectively and clarify the origin of the local maximum in the scattering curves.

Author Contributions: Conceptualization, M.S.; methodology, H.L. and J.K.; validation, J.K. and H.L.; formal analysis, J.K., H.L., M.S. and I.A.B.; investigation, H.L. and M.S.; resources, I.A.B. and H.L.; data curation, J.K. and H.L.; writing—original draft preparation, M.S. and J.K.; writing—review and editing, M.S., J.K. and H.L.; visualization, J.K. and I.A.B.; funding acquisition, M.S. All authors have read and agreed to the published version of the manuscript.

Funding: The research of I.A.B. was funded by the State Conference of Women and Equality Officers at Bavarian Universities (LaKoF Bavaria, Ph.D. scholarship). Open Access Funding by the University of Vienna.

Data Availability Statement: The data that support the findings of this study are openly available at: https: / / doi.ill.fr/10.5291/ILL-DATA.1-04-130 (accessed on 12 May 2021; registration of ILL user account required).

Acknowledgments: The authors thank Elena Kramarenko for useful discussions and Tobias Probst for assistance with preparation of experiments in Regensburg. The authors are grateful to Alexander Brunhuber and Wolfgang Kettl for providing MAE samples with stiff elastomer matrix. The authors thankfully acknowledge the anonymous reviewers for constructive criticism and useful suggestions.

Conflicts of Interest: The authors declare no conflict of interest.

\section{References}

1. Filipcsei, G.; Csetneki, I.; Szilágyi, A.; Zrínyi, M. Magnetic Field-Responsive Smart Polymer Composites. In OligomersPolymer Composites-Molecular Imprinting; Gong, B., Sanford, A.R., Ferguson, J.S., Eds.; Advances in Polymer Science; Springer: Berlin/Heidelberg, Germany, 2007; pp. 137-189. ISBN 978-3-540-46830-1.

2. Li, Y.; Li, J.; Li, W.; Du, H. A State-of-the-Art Review on Magnetorheological Elastomer Devices. Smart Mater. Struct. 2014, 23, 123001. [CrossRef]

3. Ubaidillah; Sutrisno, J.; Purwanto, A.; Mazlan, S.A. Recent Progress on Magnetorheological Solids: Materials, Fabrication, Testing, and Applications. Adv. Eng. Mater. 2015, 17, 563-597. [CrossRef]

4. Menzel, A.M. Tuned, Driven, and Active Soft Matter. Phys. Rep. 2015, 554, 1-45. [CrossRef]

5. Odenbach, S. Microstructure and Rheology of Magnetic Hybrid Materials. Arch. Appl. Mech. 2016, 86, 269-279. [CrossRef]

6. López-López, M.; Durán, J.; Iskakova, L.; Zubarev, A. Mechanics of Magnetopolymer Composites: A Review. J. Nanofluids 2016, 5, 479-495. [CrossRef]

7. Weeber, R.; Hermes, M.; Schmidt, A.M.; Holm, C. Polymer Architecture of Magnetic Gels: A Review. J. Phys. Condens. Matter 2018, 30, 063002. [CrossRef]

8. Shamonin, M.; Kramarenko, E.Y. Chapter 7-Highly Responsive Magnetoactive Elastomers. In Novel Magnetic Nanostructures; Domracheva, N., Caporali, M., Rentschler, E., Eds.; Advanced Nanomaterials; Elsevier: Amsterdam, The Netherlands, 2018; pp. 221-245. ISBN 978-0-12-813594-5.

9. Bastola, A.K.; Paudel, M.; Li, L.; Li, W. Recent Progress of Magnetorheological Elastomers: A Review. Smart Mater. Struct. 2020, 29, 123002. [CrossRef]

10. Cantera, M.A.; Behrooz, M.; Gibson, R.F.; Gordaninejad, F. Modeling of Magneto-Mechanical Response of Magnetorheological Elastomers (MRE) and MRE-Based Systems: A Review. Smart Mater. Struct. 2017, 26, 023001. [CrossRef]

11. Abramchuk, S.; Kramarenko, E.; Stepanov, G.; Nikitin, L.V.; Filipcsei, G.; Khokhlov, A.R.; Zrínyi, M. Novel Highly Elastic Magnetic Materials for Dampers and Seals: Part I. Preparation and Characterization of the Elastic Materials. Polym. Adv. Technol. 2007, 18, 883-890. [CrossRef]

12. Borbáth, T.; Günther, S.; Borin, D.; Gundermann, T.; Odenbach, S. X $\mu$ CT Analysis of Magnetic Field-Induced Phase Transitions in Magnetorheological Elastomers. Smart Mater. Struct. 2012, 21. [CrossRef]

13. An, H.-N.; Picken, S.J.; Mendes, E. Direct Observation of Particle Rearrangement during Cyclic Stress Hardening of Magnetorheological Gels. Soft Matter 2012, 8, 11995-12001. [CrossRef] 
14. Schümann, M.; Odenbach, S. In-Situ Observation of the Particle Microstructure of Magnetorheological Elastomers in Presence of Mechanical Strain and Magnetic Fields. J. Magn. Magn. Mater. 2017, 441, 88-92. [CrossRef]

15. Gundermann, T.; Cremer, P.; Löwen, H.; Menzel, A.M.; Odenbach, S. Statistical Analysis of Magnetically Soft Particles in Magnetorheological Elastomers. Smart Mater. Struct. 2017, 26, 045012. [CrossRef]

16. Avdeev, M.V.; Aksenov, V.L. Small-Angle Neutron Scattering in Structure Research of Magnetic Fluids. Phys. Uspekhi 2010, 53, 971. [CrossRef]

17. Odenbach, S.; Schwahn, D.; Stierstadt, K. Evidence for Diffusion-Induced Convection in Ferrofluids from Small-Angle Neutron Scattering. Z. Für Phys. B Condens. Matter 1995, 96, 567-569. [CrossRef]

18. Aksenov, V.; Avdeev, M.; Balasoiu, M.; Rosta, L.; Török, G.; Vekas, L.; Bica, D.; Garamus, V.; Kohlbrecher, J. SANS Study of Concentration Effect in Magnetite/Oleic Acid/Benzene Ferrofluid. Appl. Phys. A 2002, 74, s943-s944. [CrossRef]

19. Pop, L.M.; Hilljegerdes, J.; Odenbach, S.; Wiedenmann, A. The Microstructure of Ferrofluids and Their Rheological Properties. Appl. Organomet. Chem. 2004, 18, 523-528. [CrossRef]

20. Balasoiu, M.; Craus, M.L.; Plestil, J.; Haramus, V.; Erhan, R.; Lozovan, M.; Kuklin, A.I.; Bica, I. Microstructure of Magnetite Doped Elastomers Investigated by SAXS and SANS. 2008, 11. Available online: https://www.researchgate.net/publication/242829586_ Microstructure_of_magnetite_doped_elastomers_investigated_by_SAXS_and_SANS (accessed on 12 May 2021).

21. Balasoiu, M.; Craus, M.L.; Anitas, E.M.; Bica, I.; Plestil, J.; Kuklin, A.I. Microstructure of Stomaflex Based Magnetic Elastomers. Phys. Solid State 2010, 52, 917-921. [CrossRef]

22. Balasoiu, M.; Lebedev, V.T.; Orlova, D.N.; Bica, I.; Raikher, Y.L. SANS Investigation of a Ferrofluid Based Silicone Elastomer Microstructure. J. Phys. Conf. Ser. 2012, 351, 012014. [CrossRef]

23. Balasoiu, M.; Lebedev, V.T.; Raikher, Y.L.; Bica, I.; Bunoiu, M. The Implicit Effect of Texturizing Field on the Elastic Properties of Magnetic Elastomers Revealed by SANS. J. Magn. Magn. Mater. 2017, 431, 126-129. [CrossRef]

24. Pyanzina, E.S.; Sánchez, P.A.; Cerdà, J.J.; Sintes, T.; Kantorovich, S.S. Scattering Properties and Internal Structure of Magnetic Filament Brushes. Soft Matter 2017, 13, 2590-2602. [CrossRef]

25. Borin, D.Y.; Bergmann, C.; Odenbach, S. Characterization of a Magnetic Fluid Exposed to a Shear Flow and External Magnetic Field Using Small Angle Laser Scattering. J. Magn. Magn. Mater. 2020, 497, 165959. [CrossRef]

26. Zákutná, D.; Graef, K.; Dresen, D.; Porcar, L.; Honecker, D.; Disch, S. In Situ Magnetorheological SANS Setup at Institut Laue-Langevin. Colloid Polym. Sci. 2021, 299, 281-288. [CrossRef]

27. Sorokin, V.V.; Belyaeva, I.A.; Shamonin, M.; Kramarenko, E.Y. Magnetorheological Response of Highly Filled Magnetoactive Elastomers from Perspective of Mechanical Energy Density: Fractal Aggregates above the Nanometer Scale? Phys. Rev. E 2017, 95, 062501. [CrossRef] [PubMed]

28. Belyaeva, I.A.; Kramarenko, E.Y.; Shamonin, M. Magnetodielectric Effect in Magnetoactive Elastomers: Transient Response and Hysteresis. Polymer 2017, 127, 119-128. [CrossRef]

29. Hainbuchner, M.; Villa, M.; Kroupa, G.; Bruckner, G.; Baron, M.; Amenitsch, H.; Seidl, E.; Rauch, H. The New High Resolution Ultra Small-Angle Neutron Scattering Instrument at the High Flux Reactor in Grenoble. J. Appl. Crystallogr. 2000, 33, 851-854. [CrossRef]

30. Zubarev, A.; Chirikov, D.; Borin, D.; Stepanov, G. Hysteresis of the Magnetic Properties of Soft Magnetic Gels. Soft Matter 2016, 12. [CrossRef]

31. Barrett, M.; Deschner, A.; Embs, J.P.; Rheinstädter, M.C. Chain Formation in a Magnetic Fluid under the Influence of Strong External Magnetic Fields Studied by Small Angle Neutron Scattering. Soft Matter 2011, 7, 6678-6683. [CrossRef]

32. Romeis, D.; Toshchevikov, V.; Saphiannikova, M. Elongated Micro-Structures in Magneto-Sensitive Elastomers: A Dipolar Mean Field Model. Soft Matter 2016, 12, 9364-9376. [CrossRef] [PubMed]

33. Snarskii, A.A.; Zorinets, D.; Shamonin, M.; Kalita, V.M. Theoretical Method for Calculation of Effective Properties of Composite Materials with Reconfigurable Microstructure: Electric and Magnetic Phenomena. Phys. A Stat. Mech. Appl. 2019, 535, 122467. [CrossRef]

34. Snarskii, A.A.; Shamonin, M.; Yuskevich, P.; Saveliev, D.V.; Belyaeva, I.A. Induced anisotropy in composite materials with reconfigurable microstructure: Effective medium model with movable percolation threshold. Phys. A Stat. Mech. Appl. 2020, 560, 125170. [CrossRef]

35. Pipich, V. Magnetic-Field-Induced Structural Changes in Compliant Magnetorheological Elastomers. Private Communication, 2018.

36. Stepanov, G.V.; Borin, D.Y.; Raikher, Y.L.; Melenev, P.V.; Perov, N.S. Motion of Ferroparticles Inside the Polymeric Matrix in Magnetoactive Elastomers. J. Phys. Condens Matter 2008, 20, 204121. [CrossRef] [PubMed]

37. Bodnaruk, A.V.; Brunhuber, A.; Kalita, V.M.; Kulyk, M.M.; Kurzweil, P.; Snarskii, A.A.; Lozenko, A.F.; Ryabchenko, S.M.; Shamonin, M. Magnetic Anisotropy in Magnetoactive Elastomers, Enabled by Matrix Elasticity. Polymer 2019, 162, 63-72. [CrossRef] 